\title{
Low temperature impact toughness of the main gas pipeline steel after long-term degradation
}

Research Article

\author{
Pavlo O. Maruschak ${ }^{1}$, , Iryna M. Danyliuk ${ }^{1}$, Roman T. Bishchak ${ }^{2}$, Tomaž Vuherer ${ }^{3}$ \\ 1 Ternopil Ivan Pul'uj National Technical University, 46001, Ruska 56, Ukraine \\ 2 Ivano-Frankivsk National Technical University of Oil and Gas, 76019, Karpatska 15, Ukraine \\ 3 University of Maribor, Smetanova 17, SI-2000, Slovenia
}

Received 26 March 2014; accepted 28 July 2014

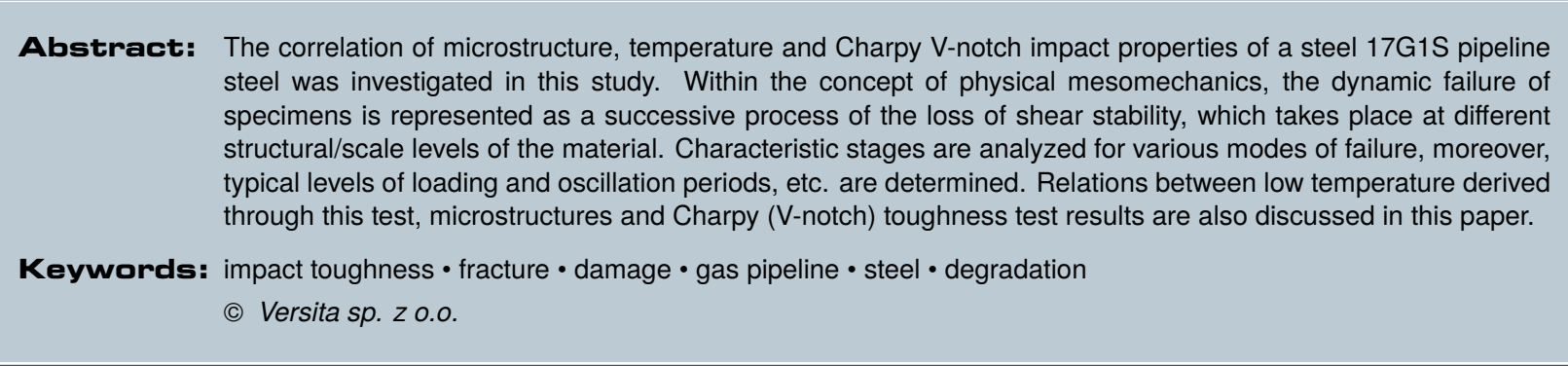

\section{Introduction}

A significant part of the main gas pipelines of Ukraine have been in operation for a long time, therefore, a comprehensive study of the structural and mechanical degradation and the factors leading to it is very topical now $[1,2]$. The operating conditions of such structures are characterized by the effect of the external force factors (loading, temperature, medium, etc.), which periodically vary with time, degradation of the physical and mechanical properties of metal with time, nucleation and propagation of defects in them $[3,4]$. These factors are crucial to the assessment of the ultimate state and predicting

*E-mail: Maruschak.tu.edu@gmail.com the residual life of the structural elements with defects (cracks). In previous works, the form and nature of the occurrence of pitting and other concentrators were investigated, which were caused by the imperfect electrochemical protection, negligence in the process of construction or repair. Another type of damage is the corrosive cracking under loading, which is caused by simultaneous effect of the corrosive medium and tensile stresses $[5,6]$. As a rule, this type of damage occurs on the external surface of the gas pipeline and has a form of multiple cracks oriented along the pipe. It is these defects that can be stress concentrators causing unpredictable failure of the film-insulated gas pipeline with diameter of 1020-1420 m [7].

The most objective investigation of the properties of pipes of the main pipelines is their full-scale pneumatic field testing. However, due to its high cost and laboriousness 


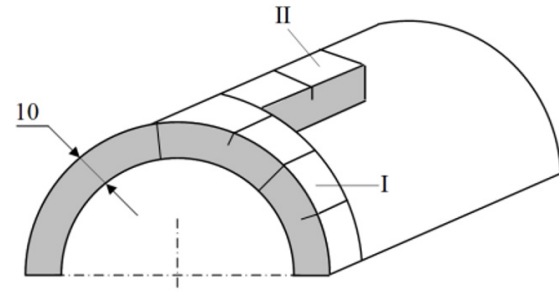

Figure 1. Schemes for specimen cutting and application of concentrators on Charpy specimens investigated.

it is not widely used. Moreover, a significant advantage of the laboratory testing is the possibility to obtain a large statistical selection of data. In addition, a correlation between the results of impact tests and field tests is being studied intensively at present [8].

The existing practice of the complex technical diagnostics and analysis of the safe operation of pipelines, reservoirs and pressure vessels does not fully (fragmentarily) take into account the said peculiarities. Therefore, there is a need to create a technique for the strength-based support of the faultless operation of the critical structural elements, which is based on the approved criteria for the evaluation of the ultimate state and can be used to predict the brittle, mixed (quasi-brittle) and ductile nature of failure. It should also take into account the actual operating conditions, presence of progressing defects, and be based on the up-to-date technologies of gathering, storage, processing and presentation of information $[9,10]$. The purpose of this work is to evaluate impact toughness of the pipe material of the main pipeline after 40 years of operation.

\section{Research technique}

Properties of the metal of the main gas pipeline "KievWest 1" were studied. The structure of the internal and external layers of the pipe were analyzed using the metallographic microscope Axiovert 40 MAT. Impact toughness was measured on Charpy specimens of size $10 \times 10 \times 55 \mathrm{~mm}$. The radius of the $V$-shaped notch was $0.25 \pm 0.025 \mathrm{~mm}$. The cutting scheme and designation of specimen types are presented in Figure 1.

Impact toughness of the material of the main gas pipeline after 40 years of operation was determined on the automated impact testing machine RKP - 300 equipped with the VUHI - CHARPY software. The program allows dividing the energy of the specimen failure on components by transforming the "loading - time" $(P-t)$ dependence into the "loading - shift" $(P-s)$ dependence. To this end, the hammer mass (loading application) $m$, and the initial impact velocity $v_{0}$ were taken into account. Considering dependence $P(t)$, the variation of the hammer displacement velocity $v(t)$ under loading was calculated using double successive integration according to recommendations [11]:

$$
v(t)=v_{0}-\frac{1}{m} \int_{t_{0}}^{t} P(t) d t
$$

After that, the dependence of hammer displacement $s$ within loading time $t$ was determined as:

$$
s(t)=\int_{t_{0}}^{t} v(t) d t
$$

After calculating the area under the obtained $P(s)$ diagrams, we determined the components of energy $E$ spent on failure of specimens at certain stages. Moreover, the total energy to failure of specimens $A$ under impact loading was considered as the sum of energies to nucleation $A_{i}$ and propagation $A_{p}$ of the crack [11]:

$$
A=A_{i}+A_{p}
$$

\section{Initial properties}

Steel of the main gas pipeline "Kiev - West of Ukraine 1" (KWU-1) (diameter $1020 \mathrm{~mm}$, wall thickness $10 \mathrm{~mm}$, rubber-bituminous insulation coating) was investigated. For the pipes with diameter of $1020 \mathrm{~mm}$, the hotrolled plate was supplied in the normalized state (normalization procedure for steel 17G1S consists of heating to temperature of $920-930^{\circ} \mathrm{C}$, holding during $1.0-$ $1.2 \mathrm{~mm} / \mathrm{min}$, and cooling in the air or under ventilator). After normalization steel 17G1S has a ferritic-pearlitic microstructure. It should be noted that the yield strength $\left(\sigma_{y s}\right)$, ultimate strength $\left(\sigma_{u s}\right)$ and strain $(\varepsilon)$ of metal of the main gas pipelines have a certain scatter, Figure 2(a). At the same time, the substructural non-uniformity of the pipe metal preconditioned by the process of their production leads to a significant scatter of the impact toughness values, Figure 2(b).

As a rule [12], the lower threshold of cold brittleness of the normalized steel $17 \mathrm{G} 1 \mathrm{~S}$ is not higher than $-90^{\circ} \mathrm{C}$, and the upper one is at the level of $-10^{\circ} \mathrm{C}$. The temperature of the upper threshold of cold brittleness for melts with a content 


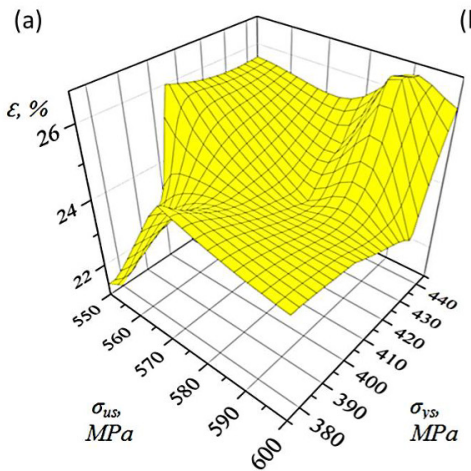

(b) $K C V_{\left(-40^{\circ} \mathrm{C}\right)}$

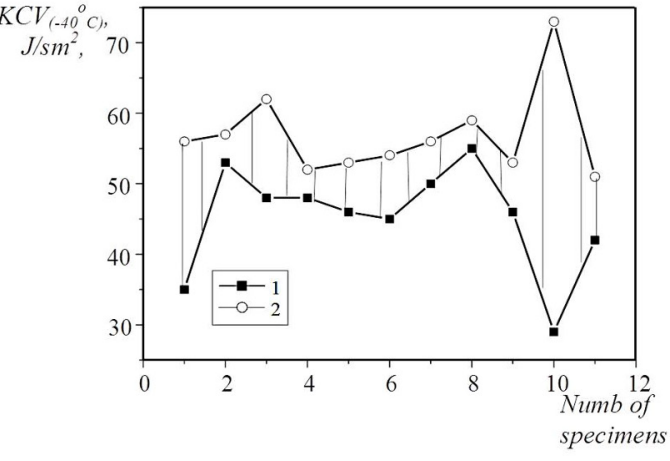

Figure 2. Initial mechanical properties (a) impact toughness of steel 17G1S (b) sets of pipes of pipeline "Kiev-West of Ukraine 1" (initial state) according to certificate 162/3-69 1,2 - upper and lower limit lines.

of carbon close to the lower limit $(0.15 \%)$ decreases to $40^{\circ} \mathrm{C}$. It should be noted that steel $17 \mathrm{G} 1 \mathrm{~S}$ is sensitive to deformational aging, due to which a decrease in impact toughness is observed [12].

\section{Fracture energy}

It is theoretically substantiated and experimentally proved that plastic deformation develops gradually in a loaded hard body, similar to the evolution of the scale levels in case of loss of shear stability. Deformation mechanisms, their carriers on the curve "effort - time" at various scale levels are described by the principle of scale invariability. Each stage is characterized by self-organization of the meso-substructure elements relevant to it, and is replaced by the stage of a higher scale level at the point of bifurcation. Failure is classified as a global loss of shear stability at the macroscale level.

It is obvious that the fracture energy in case of nucleation and propagation of the crack is determined by the ability of its structure to resist crack propagation. The dynamic crack growth resistance $J_{l d}$ of specimens was determined from formula [13]:

$$
J_{l d}=\frac{A_{i}}{B(W-a)}
$$

where $A$ is the energy to crack nucleation; $B, W$ is the width and height of the specimen; $a$ is the notch depth. The results characterizing the dependence of the impact toughness of steel 17G1S on the test temperature have been obtained. It is found that a decrease in temperature causes a decrease in the energy to crack nucleation and propagation, Table 1.
Table 1. Results of low temperature influence on impact toughness.

\begin{tabular}{lcllll}
\hline $\begin{array}{l}\text { Type of } \\
\text { specimen }\end{array}$ Temperature $A, J$ & $A_{i}, J$ & $A_{p}, J$ & $\begin{array}{l}K C V \\
\mathrm{MJ} / \mathrm{m}^{2}\end{array}$ \\
\hline \multirow{4}{*}{ I } & 20 & 36.02 & 14.64 & 21.38 & 0.45 \\
& -30 & 30.14 & 9.39 & 20.75 & 0.38 \\
& -60 & 12.56 & 1.24 & 11.32 & 0.15 \\
II & 20 & 28.03 & 4.50 & 23.53 & 0.35 \\
& -30 & 27.89 & 3.60 & 23.30 & 0.34 \\
& -60 & 8.02 & 3.20 & 4.82 & 0.11 \\
\hline
\end{tabular}

It is established that a decrease in the test temperature decreases impact toughness of the material failure, Figure 3(a). Most sensitive to low temperatures are the specimens of type $\mathrm{I}$, in which the energy to crack nucleation in case of a decrease in temperature from 20 to $-60^{\circ} \mathrm{C}$ decreases by 12 times. The fracture energy of the crack growth decreases only by 1.8 times in this case. For the specimens of type II such a decrease in temperature causes a lesser effect. In particular, the energy to crack nucleation decreases by 1.4 times, and the energy to crack propagation - by 4.1 times, Figure 3(b).

\section{Approaches of physical mesomechanics}

The approaches of physical mesomechanics allow describing the most important peculiarities of behavior of relevant materials, including the processes of localization of deformation and failure [14]. Such calculations allow determining the main structural levels of the deformation process under conditions of dynamic loading. In a number 
(a)

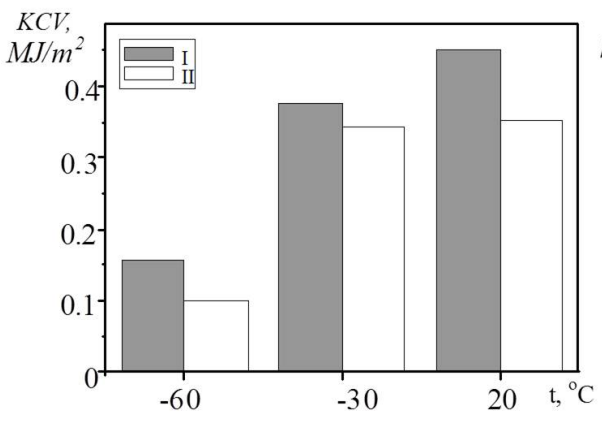

(b)

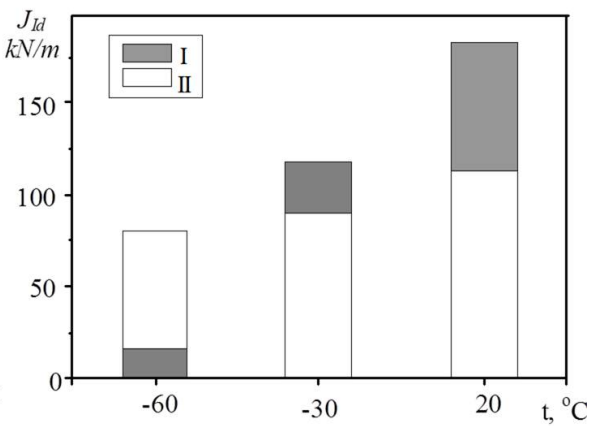

Figure 3. Dependence of impact toughness KCV (a) and $I_{I d}$ (b) on test temperature: I, II - specimen types according to Figure 1.

of cases, in order to ensure the adequate description of the deformation process of steels, taking into account their structural and mechanical peculiarities, we should consider the peculiarities of their structure, as well as the mechanism of the crack start and propagation.

In the specimen loading diagram, Figure 4(a), significant oscillations of loading during crack nucleation are traceable. Localization bands are formed in the vicinity of the notch during impact, a local zone in the vicinity of the concentrator is involved in the zone of deformation. Redistribution of strain localization bands is depicted by oscillations on the stress-strain curve. After that a separation crack is nucleated, which propagates into the specimen depth.

Strains in the vicinity of the concentrator have a significant shear component, which is depicted by several oscillations on the stress-strain curve. Deformation processes are localized in the vicinity of the concentrator. The start of a crack causes relaxation processes in the vicinity of the concentrator, and the brittle start of the crack, Figure 4(b).

On the diagram shown in Figure 4(c), we can notice the low amplitude oscillation processes connected with the formation of strain localization bands. These processes are caused by the non-uniformity of the material structure, non-uniform stress-strain state, and dynamic excitation of deformation processes due to the inflation and scatter of the impact energy. The availability of a certain non-uniformity of steels affects the kinetics of strain development in the vicinity of the notch, which causes the formation of the local hardened sections. The effect of the micro shear processes causes variation of the mechanisms of inelastic strains development, resulting in an expansion or narrowing of the localization zones.
As is seen in the "energy to failure - time" diagram, Figure 4(d), the energy to failure at 20 and $-30^{\circ} \mathrm{C}$ decreases by 1.2 times, at $-60^{\circ} \mathrm{C}$ the energy to failure decreases by 3.5 times. Moreover, the embrittling action of low temperatures manifests itself in a significant shortening of the specimen failure time as compared to normal conditions.

The nucleation of the crack took place quite smoothly, with insignificant low-amplitude oscillations, Figure 5(Đř). The presence of loading oscillations for this type of specimens was observed on the descending part of the diagram. They are preconditioned by local delaminations of the material during crack propagation. The fact that these phenomena took place on the descending part of the diagram proves that such delaminations did not exist at the moment of the crack start.

The diagram of this specimen is very interesting, Figure 5(b). The crack start took place practically without oscillations. In general, the diagram has the "orderly" shape, the loading oscillations are practically absent. An intensive macro drop in loading of high intensity found on the descending part of the diagram is connected with the crack jump and the formation of macro delamination. Further deformation of the specimen causes a significant localization and takes place due to the involvement of large volumes of the specimen in the deformation process. After that, the diagram transfers to another peak, after which the crack growth and specimen rupture take place.

The diagram has oscillations during the crack nucleation, Figure 5(c), which confirms the statements that the nucleation process is a non-linear undulatory process. The crack start is quite energy consuming, and its propagation practically does not cause relaxation processes (drop in loading). In our opinion, this is 
(a)

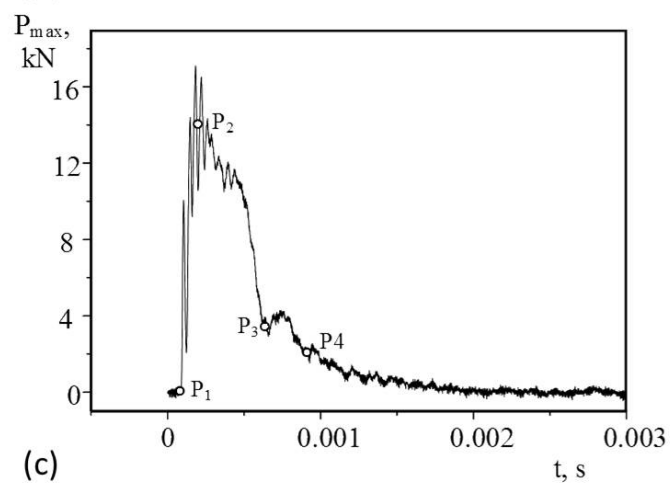

(b)

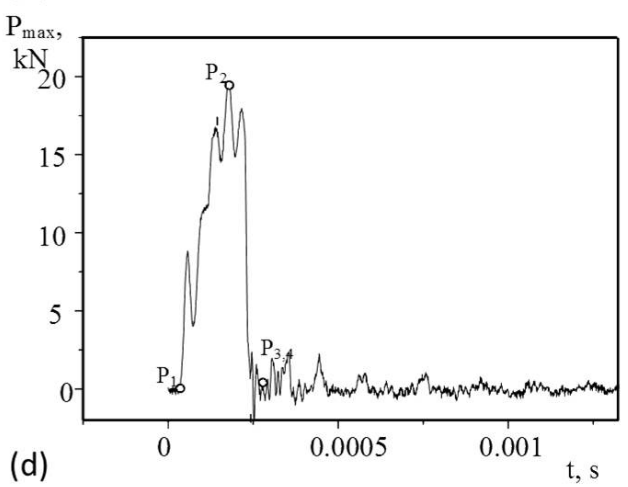

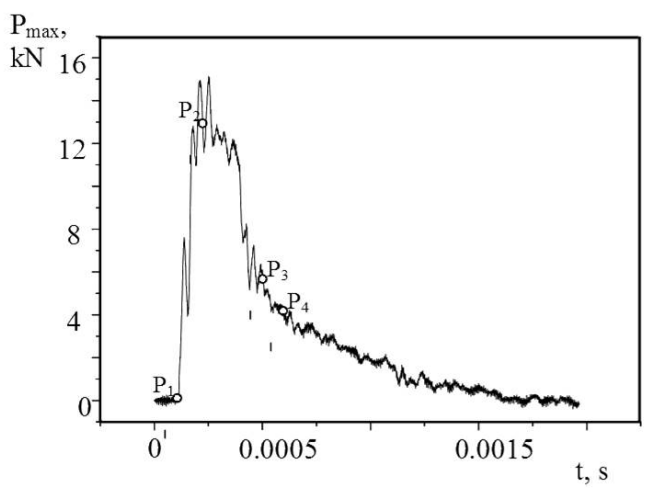

A, $\mathrm{J}$

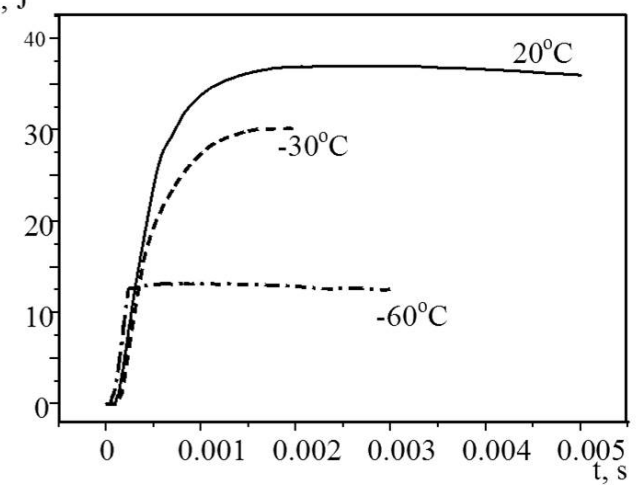

Figure 4. Dynamic influence on Charpy specimens (type I, see Figure 1) at $20^{\circ} \mathrm{C}(\mathrm{a}),-30^{\circ} \mathrm{C}(\mathrm{b}),-60^{\circ} \mathrm{C}$ (c) and variation of energy to failure with time (d).

connected with a decrease in deformation processes of the turning type. At the same time, despite the presence of the composite structure, which causes a partial arrest of the crack, low test temperatures decrease the self-ordered deformation between the bands of ferrite and pearlite. It is this that causes brittle rupture of the specimen, which is depicted by the sharp drop in loading.

The process of impact deformation is closely connected with the mechanisms of the long-term (40 years) deformational aging, which is caused by the accumulation of irreversible microplastic stresses, Figure 5(d). Diagram "energy to failure - time", Figure 5(c), shows that the energy to failure at 20 and $-30^{\circ} \mathrm{C}$ is commensurable, at $-60{ }^{\circ} \mathrm{C}$ the energy to failure decreases threefold. In addition, the embrittling action of low temperatures manifests itself in the reduced intensity of relaxation processes at the macrolevel in case of the accumulation of identical dislocations near various obstacles (boundaries between grains or subgrains, admixed atoms, vacancies).

\section{Synergetic aspects of dynamic deformation and failure}

One of the main peculiarities of the dynamic loading of materials is the extremely non-uniform distribution of strains in the specimen. The resistance of steel specimens to the nucleation and propagation of the crack was analyzed based on the impact loading diagrams. A number of high-frequency modulations, which are traditionally linked to the variation of the dislocation structure of the material and evolution of the dislocation charge [15], were observed on the stress-strain curves drawn for all the specimens investigated. It is known that the crack start is preconditioned by the local instability of properties in the form of a stream of dislocations, whereas a transition of the crack start to the crack propagation is the process of the accumulation of the critical concentration of defects in a certain volume, which, in the long run, predetermines the specimen failure process [16]. 
(a)

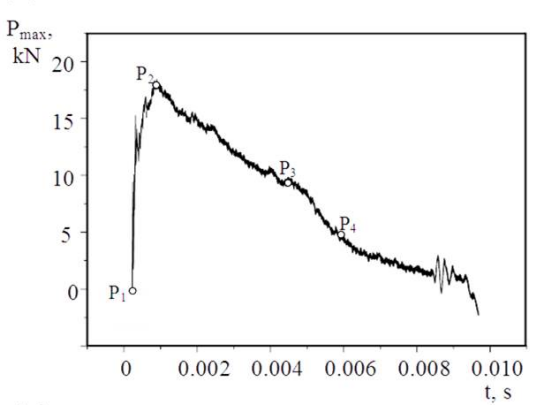

(c)

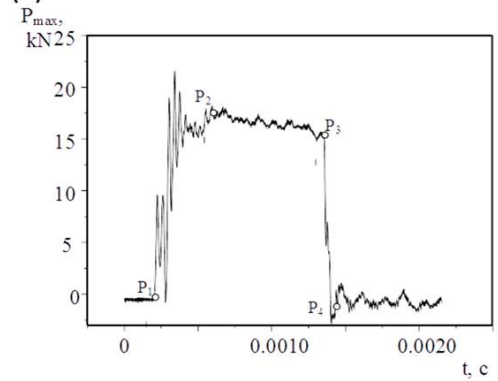

(b)

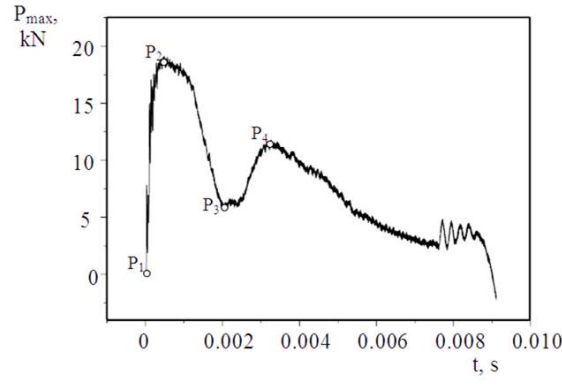

(d)

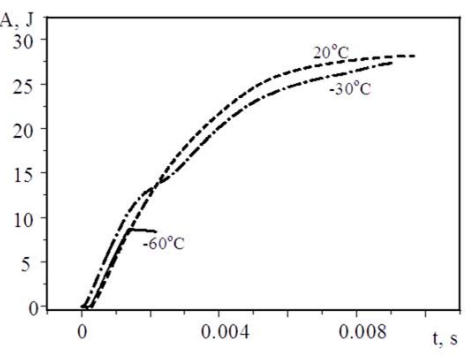

Figure 5. Dynamic influence on Charpy specimens (type II, see Figure 1) at $20^{\circ} \mathrm{C}(\mathrm{a}),-30^{\circ} \mathrm{C}(\mathrm{b}),-60^{\circ} \mathrm{C}$ (c) and variation of energy to failure with time (d)

According to the literature sources, an increase in a degree of the system non-equilibrium leads to a faster loss of its stability [17]. After passing the point of bifurcation in case of the occurrence of a new additional dissipation mechanism, it may come to a state with the asymptotic stability. Such dissipation mechanisms may occur during transition to a new scale level of the material deformation [18].

Proceeding from the above, an individual stage of deformation/failure can be characterized as the asymptotic stable state of the material, resulting from the deformational evolution of the hard body and characterized by the ultimate state of the material at a certain structural level $[16,18]$. Based on the above mentioned principles, some characteristic points of bifurcation (transitional points) were singled out on the dynamic stress-strain curve, which describe the transitional state of the material in the process of deformation. Certain values of loading under given loading conditions correspond to them.

As an example, which integrally describes the variation of the energetic state of the system under the action of the dynamic impact, it is customary to use the material deformation energy $[11,16]$. From the point of view of fracture mechanics, the above processes can be presented in the form of two stages: 1) nucleation and 2) propagation of the crack. In this case, it is presumed that the shape of the dynamic stress-strain curve depends on the fracture energy and mechanisms of each of these stages, but most influential of them is the one that is most energy efficient (the principal one).

From the point of view of synergetics and scale levels of deformation and failure, a more detailed hierarchical classification is proposed (Figure 6). The first stage $\left(P_{1}-P_{2}\right)$ is the stage of an increase in the material energy density up to the limiting value, which corresponds to the fracture energy of the crack start. The second stage $\left(P_{2}-P_{4}\right)$ corresponds to the transformation of the material scale levels according to the adaptive (deformation) and dissipative (crack growth) mechanisms. Variation of the energy to failure within this section is connected with the peculiarities of propagation of the localized shears in the material in the stress concentrator zone, which, in its turn, predetermines the fracture energy of the crack start and is depicted on the diagram. A further development of such mechanism leads to a loss of stability due to localization of plastic strains. We should point out the role of certain points and sections on the impact stress-strain curve of 


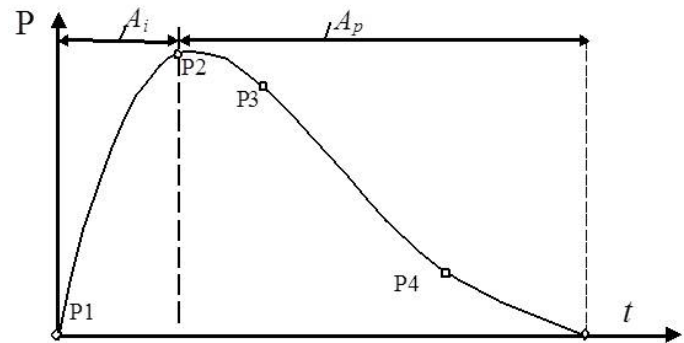

Figure 6. Structural-energetic stress-time curve for steel 17G1S: $P_{1}-P_{2}$ is micro-level of loss of shear stability (crack initiation); $P_{2}-P_{4}$ is the crack propagation

the material. Thus, the adjustment (adaptation) and selforganization of the material structure to the conditions of the external impact influence takes place within the section from $P_{1}$ to $P_{2}$ and $P_{3} ; P_{3}-P_{4}$ is the section of the beginning of the intensive failure; $P_{4}$ is the point of the minimal carrying capacity of the material;

Thus, the results obtained allow assessing the effect of the physical regularities in the material deformation and the connection between the shape of the impact stress-strain curve and stages of the material failure: $P_{1}$ is the start of loading; $P_{2}$ is the maximum carrying capacity of the material; $P_{3}$ is the beginning of the unstable failure; $P_{4}$ is blunting of the crack during failure.

\section{Conclusion}

Based on the complex use of the approaches of synergetics, physical mechanics and fracture mechanics the general regularities in deformation and failure of specimens from steel 17G1S under impact loading are formulated. It is shown that the concept of multilevel deformation and failure allows to adequately describe the variation of the energetic condition of the materials studied. Under dynamic deformation, a developed hierarchy of structural levels of plastic deformation is created in the material, which causes the self-organized plastic yielding of the entire material volume.

Within the concept of physical mesomechanics, the dynamic failure of specimens is represented as a successive process of the loss of shear stability, which takes place at different structural/scale levels of the material. Characteristic stages are analyzed for various modes of failure, moreover, typical levels of loading and oscillation periods, etc. are determined.

Points of bifurcation are singled out on the impact stress- strain curves, which in case of variation of the nature of failure may lead to a change in the mechanism of deformation and failure.

\section{References}

[1] Nykyforchyn H. M., Effect of hydrogen on the kinetics and mechanism of fatigue crack growth in structural steels, Mat Sci, 33, 1997, 504-515

[2] Zagyrski A., Matysiak H., Tsyrulnyk O. T., Zvirko I. I., Nykyforchyn H. M., Kurzydlowski K., Corrosion and stress corrosion cracking of exploited storage tank steel, Mat Sci, 40, 2004, 421-433

[3] Krasovskii A. Ya., Lokhman I. V., Orynyak I. V., Stress-corrosion failures of main pipelines, Strength of Materials, 44, 2012, 129-143

[4] Kotrechko S. O., Krasowsky A. Ya., Meshkov Yu. Ya., Torop V. M., Effect of long-term service on the tensile properties and capability of pipeline steel 17GS to resist cleavage fracture, Int J Press Vess Piping, 81, 2004, 337-344

[5] Nykyforchyn H., Lunarska E., Tsyrulnyk O. et al. Environmentally assisted 'in-bulk' steel degradation of long term service gas trunkline, Eng. Failure Analysis, 17, 2010, 624-632

[6] Majid Z. A., Mohsin R. Failure Investigation of natural gas pipeline, Arabian J for Sci. and Eng, 37, 2012, 1083-1088

[7] Pilkey A. K., Lambert S. B., Plumtree A., Stress corrosion cracking of $\mathrm{X}-60$ line pipe steel in a carbonate-bicarbonate solution, Corrosion, 51, 1995, 91-96

[8] Zong C., Zhu G., Mao W., Effect of crystallographic texture on the anisotropy of Charpy impact behavior in pipeline steel, Mater Sci Eng A, 563, 2013, 1-7

[9] Balokhonov R. R., Stefanov Yu. P., Makarov P. V., Smolin I. Yu., Deformation and fracture of surfacehardened materials at meso- and macroscale levels, Theor Appl Fracture Mech, 33, 2000, 9-16

[10] Wang W., Shan Y. Y., Yang K., Study of high strength pipeline steels with different microstructures, Mater Sci Eng A, 502, 2009, 38-44

[11] Maruschak P. O., Bishchak R. T., Vuherer T., Laws governing the dynamic fracture of two-layer bimetallic composites. Metallurgist, 55, 2011, 444449

[12] Kryzhanivs'kyi E. I., Nykyforchyn H. M., Specific features of hydrogen-induced corrosion degradation of steels of gas and oil pipelines and oil storage reservoirs, Mat Sci, 47, 2011, 127-136

[13] Botvina L. R., Blinov V. M., Tyutin M. R., Bannykh 
I. O., Blinov E. V., Fracture of high-nitrogen 05Kh20G10N3AMF steel during impact loading. Russian Metallurgy (Metally) 2012, 2012, 239-247

[14] Makarov P. V., Localized deformation and fracture of polycrystals at mesolevel, Theor. Appl. Fracture Mech., 33, 2000, 23-30

[15] Wang J. Q., Atrens A., Cousens D. R., Nockolds C., Bulcock S., Boundary Characterisation of X65 Pipeline Steel Using Analytical Electron Microscopy, J. Mater. Sci., 34, 1999, 1711-1719

[16] Balokhonov R. R., Makarov P. V., Romanova V. A.,
Smolin I. Yu., Simulation of crystal plasticity under dynamic loading, Comput Mat Sci, 16, 1999, 355-361

[17] Torop V. M., Orynyak I. V., The evaluation of structural strength of pipes and pressure vessels with axial cracks. Int J of Pressure Vessels and Piping, 53, 1993, 159-179

[18] Maruschak P., Baran D., Gliha V., A multiscale approach to deformation and fracture of heatresistant steel under static and cyclic loading. Medžiagotyra, 19, 2013, 29-33 